\title{
Vancomycin-induced severe asymptomatic immune thrombocytopenia: a rare cause
}

\author{
Yasir Ahmed MD, Christopher Sartin MD, Imran Umer MD, \\ Osama Mukarram MD, Renuka Borra MD
}

\begin{abstract}
Drug-induced immune thrombocytopenia is a challenging clinical problem that is often overlooked. Vancomycin is a rare cause of immune-mediated thrombocytopenia that can cause severe life-threatening bleeding in an acutely ill patient. The diagnosis requires a temporal relationship with the drug, exclusion of other common causes, and testing for vancomycin-induced platelet antibodies. Here we present a rare case of very severe but asymptomatic vancomycin-induced immune thrombocytopenia that resolved after discontinuation of vancomycin.
\end{abstract}

Key words: Vancomycin, thrombocytopenia, drug reaction

\section{INTRODUCTION}

Vancomycin, a glycopeptide bactericidal antibiotic, is used primarily to treat resistant Gram positive pathogens. In recent years, vancomycin use has increased dramatically secondary to increased incidence of methicillin resistant Staphylococcus aureus (MRSA) infections. ${ }^{1,2}$ Ototoxicity (especially with other ototoxic drugs) and nephrotoxicity are well known side-effects of vancomycin ${ }^{3}$; vancomycin-induced severe thrombocytopenia has rarely been reported in the literature and can cause asymptomatic laboratory abnormalities to life-threatening bleeding. Here we present a rare case of severe vancomycin-induced immune mediated thrombocytopenia without symptoms that resolved after discontinuation of vancomycin.

Corresponding author: Yasir Ahmed MD

Contact Information: Yasir.ahmed@ttuhsc.edu

DOI: 10.12746/swrccc2015.0309.119

\section{Case Presenttion}

A 63-year-old man with a history of uncontrolled diabetes mellitus presented with low grade fever and a diabetic right foot ulcer for three weeks. The rest of review of systems was unremarkable. His examination revealed temperature $101^{\circ} \mathrm{F}$, heart rate 88 beats/minute, respiratory rate 18 breaths/minute, and blood pressure $130 / 70 \mathrm{mmHg}$. Examination of the foot revealed a small, purulent ulcer with surrounding cellulitis on the planter surface of the right big toe extending to the second and third toes with normal pulses in the foot. The remainder of the physical examination was unremarkable. Magnetic resonance imaging of the right foot confirmed a small abscess in the first right toe area. He underwent debridement and drainage of the abscess, where infected tissue was found extending to the first metatarsal bone and phalanx of the toe. The patient was started on empiric vancomycin and piperacillin-tazobactam intravenously (IV). Bone tissue cultures were positive for methicillin sensitive Staphylococcus aureus (MSSA) and MRSA. 
Blood cultures at admission were positive for MSSA. Transthoracic and transesophageal echocardiograms were negative for vegetations. Repeat blood cultures on hospital day three were negative, and antibiotics were switched to IV vancomycin. The patient was discharged on hospital day seven on IV vancomycin for six weeks with wound care as an outpatient. He was advised to have a complete blood count, C-reactive protein, basic metabolic panel, and vancomycin trough levels every week.

One week later, the laboratory reported the platelet count was $2,000 / \mathrm{mm}^{3}$ (manually confirmed); it was $206,000 / \mathrm{mm}^{3}$ one week prior. The white blood cell count (WBC) was $1,200 / \mathrm{mm}^{3}$ with $60 \%$ lymphocytes, $4 \%$ eosinophils, and $8 \%$ neutrophils; one week prior the WBC was $7,800 / \mathrm{mm}^{3}$. He was immediately readmitted into the hospital. He denied bleeding, hemoptysis, melena, hematochezia, fever, skin rash, or worsening of his lower extremity pain. Other tests included hemoglobin $9.4 \mathrm{~g} / \mathrm{dl}$, peripheral smears with normal platelet morphology with no clumping, prothrombin time 11.3 second (normal 11.2-14s), activated prothrombin time 28.5 second (normal 21.4-33.1s), total bilirubin $1.2 \mathrm{mg} / \mathrm{dl}$, serum creatinine $1.2 \mathrm{mg} / \mathrm{dl}$, and vancomycin level of $16.3 \mu \mathrm{g} / \mathrm{mL}$. The heparin associated antibody was 1.159 OD (normal <0.399), but the serotonin release assay test was negative.

Vancomycin was immediately stopped, and IV daptomycin and empiric meropenem were added. His acute viral hepatitis panel, serum haptoglobin, serum lactate dehydrogenase level, anti-nuclear antibody (IgG), serum B12 level, parvovirus B19 IgM, parvovirus $\mathrm{B} 19$ by $\mathrm{PCR}$, Epstein-Barr antibody viral-capsid antigen IgM, and flow cytometry results were all negative. The patient received single donor platelets transfusions for three consecutive days as preemptive therapy. Filgrastim (human granulocyte colonystimulating factor) injection was given for four days, and his neutropenia resolved in 72 hours. Vancomycin-induced immune platelet IgG antibody was positive. The patient's platelet count improved to $80,000 /$ $\mathrm{mm}^{3}$ on day six and returned to baseline 213,000 / $\mathrm{mm}^{3}$ on day 10 after discontinuation of vancomycin. Neutropenia was probably related to partial bone mar- row suppression due to vancomycin, and the WBC count normalized to $6,500 / \mathrm{mm}^{3}$ after discontinuation of vancomycin. The patient completed four weeks of daptomycin therapy as an outpatient with no relapse of thrombocytopenia or leukopenia.

\section{Discussion}

Drug-induced immune thrombocytopenia is a challenging clinical problem that is often overlooked. ${ }^{4}$ The incidence of drug-induced thrombocytopenia is not well defined in the medical literature. Based on several reports from the United States and Europe, the estimated incidence is around 10 cases per million population per year, but the number could be higher in hospitalized or elderly patients.

Drug-induced thrombocytopenia typically results from either non-immune or immune mediated mechanisms. Non-immune thrombocytopenia is common and results from the suppression of platelet production by general myelotoxicity (e.g., chemotherapy agents), dose-dependent myelosuppression (e.g., linezolid), or interference with specific megakaryocyte function (e.g., bortezomib). Immune-mediated thrombocytopenia results from platelet destruction by drug-dependent platelet antibodies in the circulation. Several mechanisms have been proposed for immune-mediated thrombocytopenia, including: a) classic drug-dependent platelet antibodies (e.g., quinine); b) hapten-induced antibodies (e.g., penicillin); c) fiban-dependent antibodies (e.g., tirofiban); d) Fabbinding monoclonal antibodies (e.g., abciximab); e) drug-induced autoantibody formation (e.g., gold); f) immune complex formation (e.g., heparin).

Vancomycin-induced thrombocytopenia is postulated to be mediated by vancomycin-dependent immunoglobulin antibodies that bind specifically to platelet glycoprotein IIb and/ or IIla and lead to platelet destruction. The antibodies formed in the presence of vancomycin appear to act like antibodies induced by quinine. $^{7,8}$ Drygalski et al reported a large case series of vancomycin-induced immune thrombocytopenia. Vancomycin dependent, platelet-reactive 
antibodies in the immunoglobulin $\mathrm{G}$ class, immunoglobulin M class, or both were identified in 34 patients $(20 \%)$. The mean nadir platelet count was $13,600 /$ $\mathrm{mm}^{3}$, and severe bleeding occurred in $10(34 \%)$ cases. Platelet counts returned to baseline in all surviving patients after discontinuing vancomycin.

Early diagnosis of vancomycin-induced immune thrombocytopenia is essential to avoid life threatening bleeding. The diagnosis requires exclusion of common causes of thrombocytopenia, especially disseminated intravascular coagulation (DIC), a variety of infectious diseases that potentially cause thrombocytopenia, drugs, immunologic disorders, and hematologic malignancies, and a temporal relationship of the drug. The presence of vancomycininduced immune platelet antibody and resolution of thrombocytopenia after discontinuing the vancomycin confirms the diagnosis in most cases. The main treatment is to stop vancomycin and avoid future use. In most cases, platelet count will recover promptly. The role of IV immunoglobulin (IgG) is unclear but can be used in patients with severe bleeding.

Our patient had asymptomatic vancomycininduced severe immune thrombocytopenia; his platelet count dropped to $2,000 / \mathrm{mm}^{3}$ from his baseline $204,000 / \mathrm{mm}^{3}$ on the 15th day of vancomycin administration. Vancomycin was changed to daptomycin. After excluding other common causes, the diagnosis was confirmed by the presence of vancomycininduced immune platelet IgG antibodies (tests were performed at a reference laboratory, Blood Center of the Wisconsin Platelet and Neutrophil Immunology laboratory, Milwaukee, $\mathrm{WI}$ ) by using immunofluorescence flow cytometry and resolution of thrombocytopenia after discontinuation of vancomycin. Our patient had very severe thrombocytopenia, and this degree of thrombocytopenia without symptoms has been rarely documented in the literature. Mizon et al reported a case with vancomycin-induced severe thrombocytopenia with a platelet count of $2,000 / \mathrm{mm}^{3}$ with positive drug dependent platelet antibody in a patient with Staphylococcus aureus bacteremia complicated by infective endocarditis. Thrombocytopenia was resolved after discontinuation of vancomycin. ${ }^{11}$ Our patient received preemptive single donor platelets transfusion therapy for three consecutive days before the vancomycin IgG antibody test came back positive; the platelet count returned to baseline on day 10 after stopping vancomycin. He went home on IV daptomycin to complete the remaining course for right foot osteomyelitis treatment. Vancomycin rechallenge was not done due to very severe thrombocytopenia with initial therapy and confirmation of diagnosis by presence of vancomycin-induced immune platelet antibody.

In conclusion, vancomycin-induced severe immune thrombocytopenia has rarely been reported as a cause of thrombocytopenia. Physicians should monitor complete blood count and renal function closely in patients on vancomycin. Testing for vancomycin-induced immune platelet antibodies can be helpful in early diagnosis. Prompt resolution of thrombocytopenia occurs if the diagnosis is made in a timely manner, and vancomycin is discontinued.

\footnotetext{
Author Affiliation: All authors except Renuka Borra work in the Department of Internal Medicine at Texas Tech University Health Sciences Center in Odessa, TX. Christopher Sartin, Imran Umer, and Osama Mukarram are residents in internal medicine; Yasir Ahmed is a faculty member in infectious disease. Renuka Borra is a clinical assistant professor in hematology-oncology in Odessa, TX.

Received: 09/29/2014

Accepted: 12/05/2014

Reviewers: Richard Winn MD

Published electronically: 01/15/2015

Conflict of Interest Disclosures: none
}

\section{REFERENCES}

1. Michel M, Gutmann L. MRSA and VRE-therapeutic realities and possibilities. Lancet 1997; 349:1901-6. 
2. Rahman M. Alternatives to vancomycin in treating methicillin-resistant Staphylococcus aureus infections. J Antimicrob Chemother 1998; 41:325-8.

3. Levine DP. Vancomycin: a history. Clin Infect Dis 2006 Jan 1; 42 Suppl 1:S5-12.

4. Reese JA, Li X, Hauben M, Aster RH, Bougie DW, Curtis BR, George JN, Vesely SK. Identifying drugs that cause acute thrombocytopenia: an analysis using 3 distinct methods. Blood 2010 Sep 23; 116(12):2127-33. doi: 10.1182/blood-2010-03-276691. Epub 2010 Jun 8.

5. Van den Bemt PM, Meyboom RH, Egberts AC. Drug-induced immune thrombocytopenia. Drug Saf 2004; 27(15):1243-52.

6. Arnold DM, Nazi I, Warkentin TE, Smith JW, Toltl LJ, George JN, Kelton JG. Approach to the diagnosis and management of drug-induced immune thrombocytopenia. Transfusion Med Rev 2013 Jul; 27(3):137-45. doi: 10.1016/j.tmrv.2013.05.005. Epub 2013 Jul 8.

7. Von Drygalski A, Curtis BR, Bougie DW, McFarland JG, Ahl S, Limbu I, Baker KR, Aster RH. Vancomycin-induced immune thrombocytopenia. N Engl J Med 2007; 356:904-10.

8. Visentin GP, Newman PJ, Aster RH. Characteristics of quinine and quinidine-induced antibodies specific for platelet glycoprotein IIb and IIIa. Blood 1991; 77:2668-76.

9. Rowland SP, Rankin I, Sheth H. Vancomycin-induced thrombocytopenia in a patient with severe pancreatitis. BMJ Case Rep 2013. doi: 10.1136/bcr-2013-200830.

10. Ray JB, Brereton WF, Nullet FR. Intravenous immune globulin for the treatment of presumed quinidine-induced thrombocytopenia. DICP 1990 Jul-Aug; 24(7-8):693-5.

11. Mizon P, Kiefel V, Mannessier L, Mueller-Eckhardt C, Goudem and J. Thrombocytopenia induced by vancomycin-dependent platelet antibody. Vox Sang 1997; 73:49-51. 